\title{
Use and Analysis of Color Models in Image Processing
}

\author{
Bhubneshwar Sharma* and Rupali Nayyer
}

Department of Electronics and Communication Engineering, S.S.C.E.T, Punjab Technical University, India

\begin{abstract}
The use of color image processing is divided by two factors. First color is used in object identification and simplifies extraction from a scene and color is powerful descriptor. Second, humans can use thousands of color shades and intensities. Color Image Processing is divided into two areas full color and pseudo-color processing. In this processing various color models are used that are based on color recognition, color components etc. A few papers on various applications such as lane detection, face detection, fruit quality evaluation etc based on these color models have been published. A survey on widely used models RGB, HSI, HSV, $\mathrm{RGI}$, etc. is represented in this paper.
\end{abstract}

Keywords: Image processing; Color models; RGB; HIS; HSV; RGI

\section{Introduction}

An image may be defined as a two dimensional function, $f(x, y)$, where $x$ and $y$ are spatial coordinates and the amplitude of $f$ at any pair of coordinates $(x, y)$ is called the intensity or gray level of the image at that point. When $x, y$, and the intensity values of $f$ are all finite, discrete quantities, we call the image a digital image. The field of digital image processing refers to processing digital images by means of a digital computer [1]. Digital Image Processing used in various applications in information and technical systems such as Radar Tracking, Communications, television, astronomy etc. Various method involve in Digital Image Processing such as edge detection, segmentation, enhancement, smoothing and sharpening. It gives a theory in which states that any color can be produced by mixing three primary colors C1, C2, C3 at appropriate percentages. Initially, these methods were designed especially for gray scale image processing $[2,3]$. The RGB color model is standard design of computer graphics systems is not ideal for all of its applications. The red, green, and blue color components are highly correlated. This makes it is difficult to execute the image processing algorithms. Many processing techniques work on the intensity component of an image only. These processes are standard implemented using the HSI color model.

\section{Colour models}

To utilize color as a visual cue in multimedia, image processing, graphics and computer vision applications, an appropriate method for representing the color signal is needed. The different color specification systems or color models address this need. Color spaces provide a rational method to specify order, manipulate and effectively display the object colors taken into consideration. Thus the selected color model should be well suited to address the problem's statement and solution. The process of selecting the best color representation involves knowing how color signals are generated and what information is needed from these signals. In particular, the color models may be used to define colors, discriminate between colors, judge similarity between colors and indentify color categories for a number of applications. Color model literature can be found in the domain of modern sciences, such as physics, engineering, artificial intelligence, and computer science.

RGB and CMY model: Susstrunk, Sabine et al. [4] describes the specifications and usage of standard RGB color spaces promoted today by standard bodies and/or the imaging industry. There are some RGB color space implementation issues like sensor, unrendered, rendered or output color spaces, gamut size, encoding, compression, color space conversion etc. A remarkable property of this representation is that for matter surfaces, while ignoring ambient light, normalized RGB is invariant (under certain assumptions) to changes of surface orientation relatively to the light source. This, together with the transformation simplicity helped this color space to gain popularity among the researchers [5-7] (Figure 1).

HSI model: HSI model is proposed to improve the RGB model. The Hue Saturation Intensity (HSI) color model closely resembles the color sensing properties of human vision. To formula that converts from RGB to HSI or back is more complicated than with other color models. $\mathrm{Li}$, Jian-Feng et al. [5] formulated a new formula for saturation in RGBto-HSI conversion is proposed on the basis of HSI Color Space, aimed to provide more rapidity of computing in real-time control system due to fewer operations needed. The results of comparison between two conversion equations demonstrate that new conversion equation has significant advantages over traditional conversion in aspects of less operation needed in computing. But again conversion time is more. Tsung-Ying Sun et al. [8] proposed a new method using HSI color model for lane-marking detection, HSILMD, is proposed. In HSILMD, full color images are converted into HSI color representation, within the

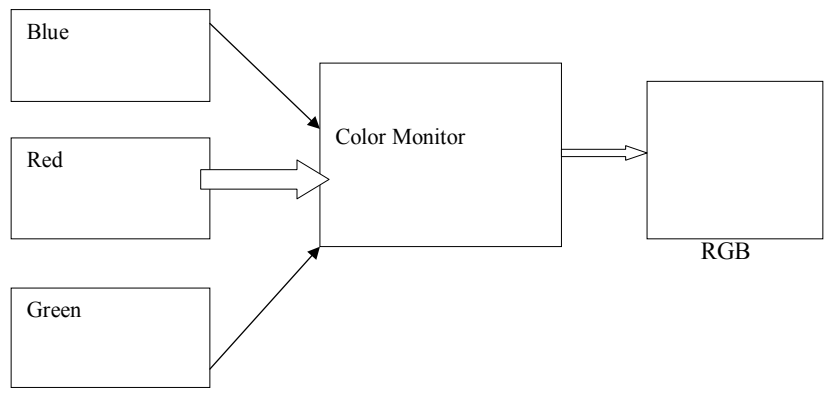

Figure 1: Generating the RGB image of the cross-sectional color plane.

*Corresonding author: Bhubneshwar Sharma, Assistant Professor, Department of Electronics and Communication Engineering, S.S.C.E.T, Punjab Technical University, India, Tel: 08054132530; E-mail: bhubnesh86@gmail.com

Received October 06, 2015; Accepted November 04, 2015; Published November 16, 2015

Citation: Sharma B, Nayyer R (2015) Use and Analysis of Color Models in Image Processing. J Food Process Technol 7: 533. doi:10.4172/2157-7110.1000533

Copyright: $\odot 2015$ Sharma B, et al. This is an open-access article distributed under the terms of the Creative Commons Attribution License, which permits unrestricted use, distribution, and reproduction in any medium, provided the original author and source are credited. 
Citation: Sharma B, Nayyer R (2015) Use and Analysis of Color Models in Image Processing. J Food Process Technol 7: 533. doi:10.4172/21577110.1000533

region of interest (ROI) aiming to detect road surface on host vehicle, with Fuzzy c-Means algorithm. Thresholds of intensity and saturation are selected accordingly. Results are compared with the same scheme using RGB color model and a different scheme

\section{Conclusion}

A survey on various color models, their description, comparison and evaluation results is presented. These models used various components of an image to display on specific hardware platform. The purpose of a color model is to facilitate the specification of colors in some standard generally accepted way. Research work also shows the conversions of various models to speed up the image processing with least time delays. But there is invariance in results of various models due to complex mathematical equations. In future, various image processing methods i.e., adaptive histogram equalization and contrast limited adaptive histogram equalization can be used to speed up the image processing by using these color models.

\section{References}

1. Gonzalez RC, Woods RE (1992) Digital Image Processing, Reading. AddisonWesley, Massachusetts.

2. Van der HF (1994) Image Based Measurement Systems. Wiley Online.

3. Castleman KR (1996) Digital Image Processing. Englewood Cliffs, PrenticeHall, New Jersey.

4. Susstrunk, Sabine, Robert Buckley, Steve Swen (1999) Standard RGB colo spaces. Color and Imaging Conference, Society for Imaging Science and Technology.

5. Li, Jian-Feng, Kaun-Quan Wang, David Zhang (2002) A new equation of saturation in RGB-to-HSI conversion for more rapidity of computing. Machine Learning and Cybernetics 3: 1493-1497.

6. Granier, Xavier, Wolfgang Heidrich (2003) A simple layered RGB BRDF model. Graphical Models 65: 171-184.

7. Wen, Che-Yen, Chun-Ming Chou (2004) Color Image Models and Its Applications to Document Examination. Forensic Science Journal 3: 23-32.

8. Harmeet Kaur kelda (2014) A Review: Color Models in Image Processing. Int J Computer Technology and Applications 5: 319-322. 